\title{
Publisher Correction: Repurposing an adenine riboswitch into a fluorogenic imaging and sensing tag
}

Sourav Kumar Dey (1), Grigory S. Filonov, Anthony O. Olarerin-George, Benjamin T. Jackson, Lydia W. S. Finley (1) and Samie R. Jaffrey (1)

Correction to: Nature Chemical Biology https://doi.org/10.1038/s41589-021-00925-0, published online 22 December 2021.

In the version of this article initially published, a processing error led to a number of pre-publication edits being overlooked. Figure $5 \mathrm{a}, \mathrm{d}$ panels are now updated to correct a calculation error affecting the presentation of the calibration bars (originally there were two colors for number "2"). Extended Data Figs. 9, 10 have been updated to now include calibration bars, and their legends lightly expanded for clarity. Refs. 12, 18, 20, 22, 31 and 41 initially had bibliographic errors and have been updated, while ref. 50 is now included in the refs. list (50. Endoh, T. \& Sugimoto, N. Selection of RNAs for constructing 'Lighting-UP' biomolecular switches in response to specific small molecules. PLoS One 8, e60222 (2013)). Further, the Supplementary Information file has been updated to correct a number of small errors in tables and figure legends.

The html and PDF versions of the article have been updated.

Published online: 21 January 2022

https://doi.org/10.1038/s41589-022-00969-w

(c) The Author(s), under exclusive licence to Springer Nature America, Inc. 2022 